\title{
PROBABLE NUEVO TEXTO CELTIBÉRICO CON SEGoBIRIGeA
}

\author{
PROBABLY A NEW CELTIBERIAN TEXT WITH SEGoBIRIGeA
}

\author{
XAVERIO BALLESTER ${ }^{1}$ \\ Universitat de València
}

MAX TURIEL

La pieza que aquí comentaremos, fue localizada por Max Turiel en una colección particular; persuadido de la relevancia de la pieza en virtud de las informaciones recibidas, gracias a diversas gestiones suyas pudo obtenerse autorización para realizar las fotografías que aquí presentamos y que, aunque ni en cantidad ni en calidad puedan, por supuesto, considerarse ideales ni profesionales, sí permitirán al menos un conocimiento suficiente del documento y del texto contenido en él. Naturalmente, a falta de la debida inspección ocular de la pieza o de los pertinentes análisis metalográficos, no puede garantizarse de manera firme la autenticidad de la misma, si bien en principio ninguno de nosotros ha detectado detalle que pueda suscitar sospechas de falsificación.

\section{DESCRIPCIÓN}

La pieza mide unos 5 centímetros de longitud - de la cabeza del ave hasta abajo- y unos 3 centímetros de anchura; es, por tanto, muy pequeña, sin embargo, está dentro de las dimensiones habituales para este tipo de documentos, que - anticipemos ya - tiene toda la apariencia de tratarse de un ejemplar de lo que tradicionalmente conocemos bajo la rúbrica de téseras de hospitalidad celtibéricas.

Tésera, así pues, de hospitalidad de bronce en forma de ave, aparentemente de una paloma; ofrece forma muy curvilínea, de estilo y tradición lateniense. La parte superior, redondeada, constituye la cabeza, ocupada por un gran ojo circular en cada uno de los lados

1. Queremos agradecer al Prof. Martín Almagro-Gorbea las observaciones tocantes a aspectos arqueológicos, históricos y tipológicos de la pieza estudiada y el habernos confirmado su procedencia del municipio conquense de Torrejoncillo del Rey. Este trabajo se ha beneficiado de una ayuda del Ministerio de Educación y Ciencia (FFI2008-01759) para el proyecto P.L.M. II, dirigido por el Prof. Dr. J. Luis Vidal (Universidad de Barcelona). y con un pico saliente, mientras que la parte final, que corresponde a la cola, ofrece una terminación plana, sin excluir que pudiera estar incompleta.

La tésera es plano-convexa. La parte convexa que cabe considerar como 'anverso' sugiere la curvatura volumétrica del cuerpo, mientras que la otra cara del 'reverso' es plana, como si el cuerpo original se hubiera cortado en dos mitades.

La parte lateral del anverso ofrece desde la cabeza a la parte del vientre una serie de 12 trazos curvados paralelos como posible esquematización del plumaje. La cara del 'reverso' es plana y muestra una inscripción hecha a base de puntos circulares incisos (vide infra), realizada desde la parte de la cola hacia la de la cabeza.

Los trazos curvados, el dorso y el fondo de los puntos de la inscripción ofrecen todavía una concreción caliza que contribuye a confirmar la impresión general de que esta pieza procede de un yacimiento arqueológico.

\section{PROCEDENCIA}

Según la información obtenida, la tésera procedería de una villa romana en Torrejoncillo del Rey, población situada en la comarca del Campo, en la provincia de Cuenca. Torrejoncillo queda en línea recta a $10 \mathrm{~km}$ al este de Carrascosa del Campo y a unos $8 \mathrm{~km}$ al Norte de Palomares del Campo, términos municipales bien conocidos por sus hallazgos arqueológicos de época celtibérica y romana. Además, El Campo es una comarca cerealista situada al norte de Segobriga, de la que Torrejoncillo del Rey queda a escasos $25 \mathrm{~km}$ en línea recta en dirección noreste, dentro por tanto de su supuesto territorium (véase Almagro-Gorbea y Lorrio, 2006-2007). Además, en la zona de Torrejoncillo, en efecto, se hallaron en su día restos arqueológicos de un «complejo minero para extraer lapis specularis o espejuelo en la Cueva de la Mora Encantada» (Sánchez-Palencia, 1993, 224), información coincidente 


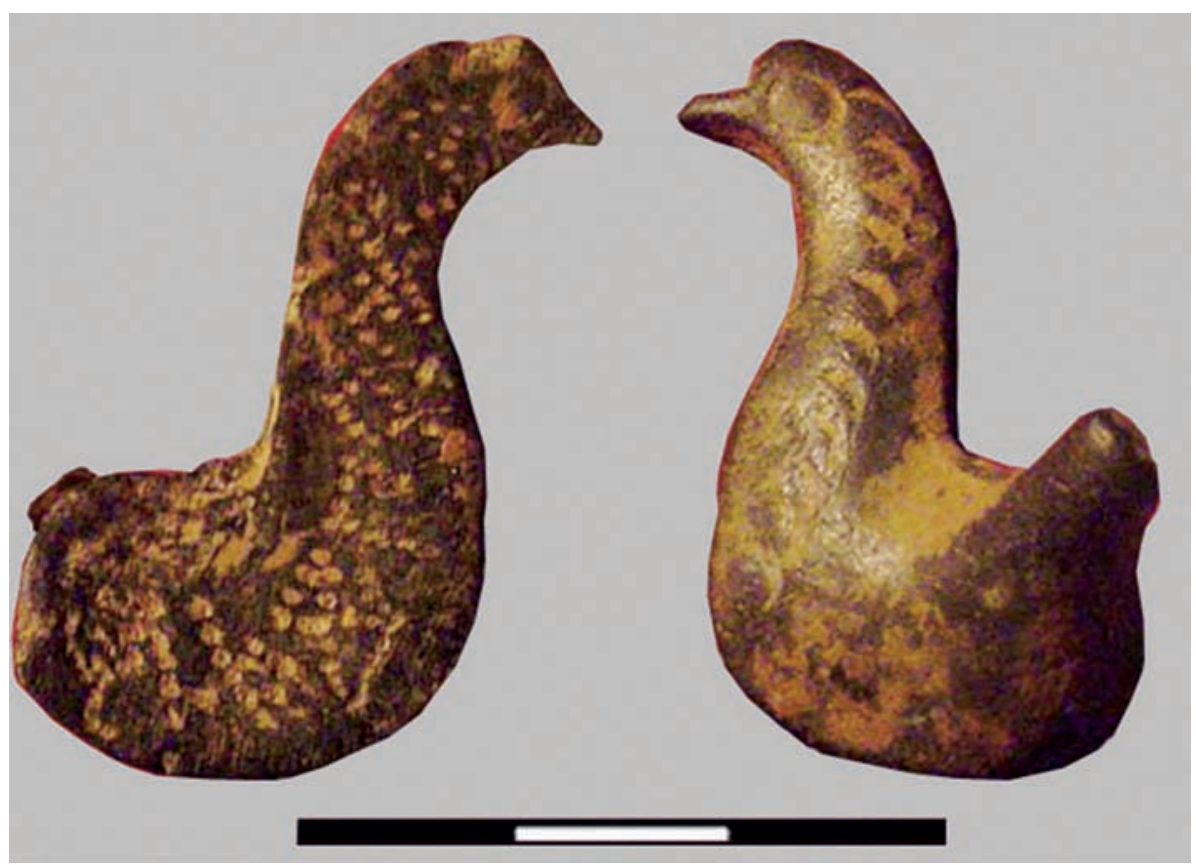

Figura 1: Tésera de Torrejoncillo del Rey.

con la que diera el tan fiable Plinio, quien precisamente señala esa zona como la mejor productora de espejuelos (nat. 36,45,160: specularis uero [...] Hispania hunc tantum Citerior olim dabat nec tota sed intra $\in$ passuum circa Segobrigam urbem; iam et Cypros et Cappadocia et Sicilia et nuper inuentum Africa. Postferendos tamen omnes Hispanice) dando un radio de cien mil pasos - unos $148 \mathrm{~km}$ - en torno a Segóbriga, localidad bien conocida por la riqueza de restos arqueológicos de naturaleza celtibérica y romana conservados (véase Almagro-Gorbea y Abascal, 1999).

\section{TIPOLOGÍA}

La pieza, como anticipábamos, presenta claramente las características más usuales en material, forma y dimensiones de las téseras celtibéricas de aspecto figurativo o documentos portátiles de hospitalidad de los pueblos celtofónicos del interior de la Península Ibérica, documentos que cuantitativamente siguen constituyendo junto con el material numismático más abundante pero lingüísticamente menos locuazel componente cuantitativamente mayor de los textos celtibéricos que actualmente conservamos.

\subsection{FORMA}

La pieza responde al modelo de tésera epígrafa, pues provista de texto, frente al modelo anepígrafo que o bien no presenta texto alguno (Almagro-Gorbea, 2003, 375-377 y 400-403) o bien presentaría marcas identificativas distintivas pero no interpretables, por cuanto hoy sabemos, como signos lingüísticos. Dentro del modelo epígrafo, nuestra pieza se inscribe en el hipótipo o subclase de texto en lengua y escritura celtibérica frente a los escritos en latín o los escritos en celtibérico mediante escritura latina. El procedimiento de inscripción responde a la igualmente también muy característica técnica de secuencia por punteado o punción, vistosa y aparentemente cómoda en superficies duras.

En cuanto a su aspecto, la pieza en concreto, como se anticipó, tiene aspecto columbiforme, representando, sin duda, un ave y ofreciendo similitud con otra pieza procedente de Palenzuela (Palencia) con inscripción sinistrorsa - id est, de derecha a izquierda - rezando UIROUIACa CaR (K.25.1 en la catalogación de Untermann, 1997, 713-714). También la pieza aquí presentada se puede relacionar con otra tésera en forma de ave-pierna de la Real Academia de la Historia (Almagro-Gorbea, 2003, 398-399, CP-17), que documentaría el uso no excepcional de las téseras aviformes. Nuestra pieza, sin embargo, tiene un carácter mucho menos biplano, proyectándose espacialmente lo que representaría la cola del animal. Como es lo más habitual en estos casos, mientras que una cara ofrece las características zoomórficas recién descritas, la otra cara del documento presenta una superficie lisa sobre la que se ha escrito el mensaje.

\subsection{DATACIÓN}

La falta de contexto arqueológico conocido impide precisar la cronología de esta tésera. Sin embargo, sus paralelos formales y epigráficos, así como el uso de 


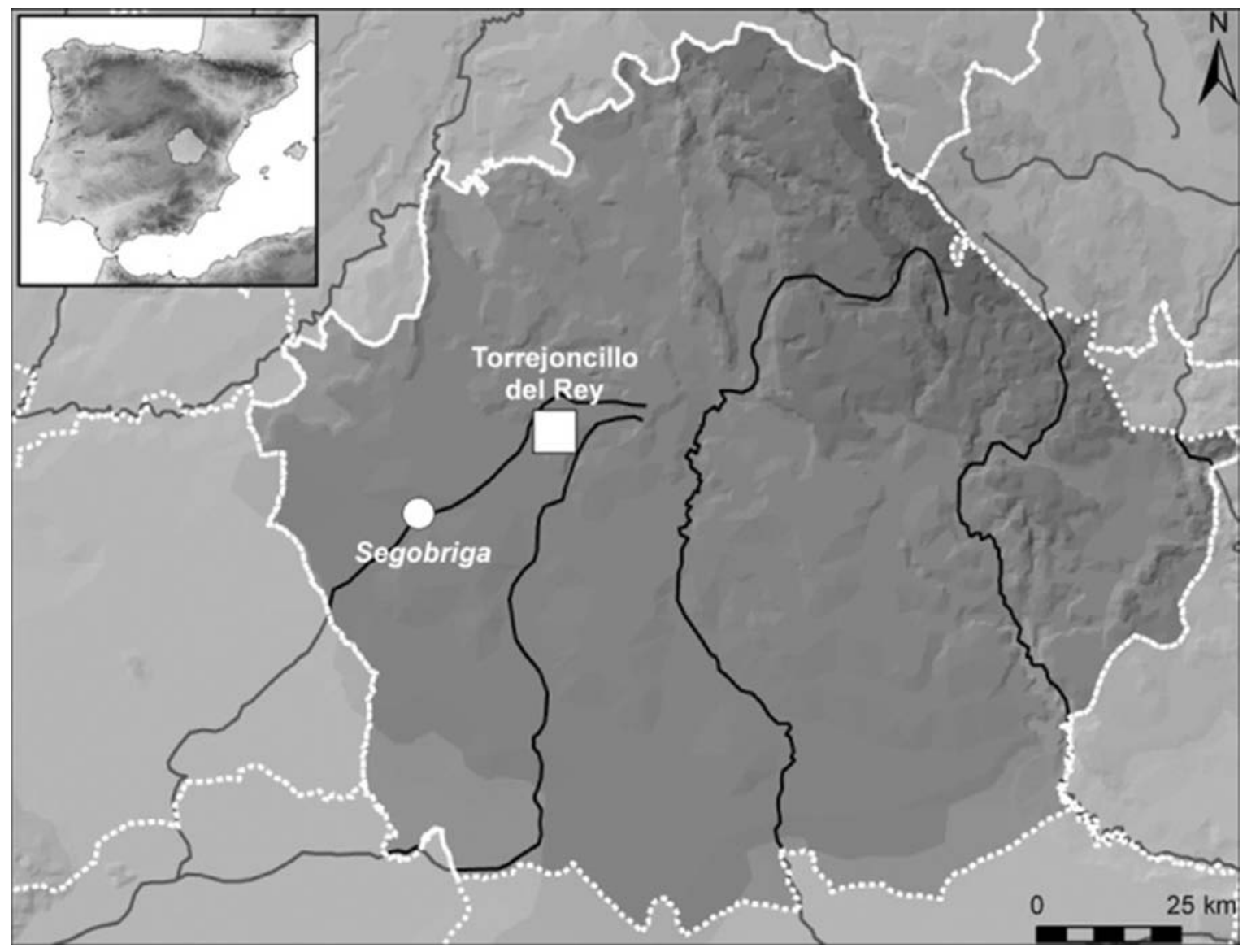

Figura 2: Lugar de hallazgo de la pieza y su relación espacial con la ciudad de Segobriga.

escritura celtibérica punteada, no latina, y su proximidad a Segobriga (cf. Almagro-Gorbea, 2003, 209212, no 103-104, 383-384 CP-3, 390-391 CP-9, etc.) permiten atribuirle una cronología a partir de fines del siglo II a.C., aunque con más probabilidad ya en la primera mitad del siglo I a.C., quizá en el contexto histórico y cultural segobrigense comprendido entre las guerras de Sertorio y las guerras civiles (vide Almagro-Gorbea y Lorrio, 2006-2007).

Reiteremos, ahora con más argumentos, que, excepto por su origen desconocido y no reglado, la pieza no ofrece ningún detalle que despierte sospechas de haber sido manipulada o falsificada. Igualmente, como enseguida veremos, la inscripción reproduce también los patrones habituales de las téseras celtibéricas manteniendo las mismas pautas de normalidad o hasta de banalidad desde el punto de vista tipológico. Podría definirse, por tanto, el presente documento como la clásica tésera celtibérica.

\section{TEXTO}

El texto se lee con dificultad pero sobre todo la secuencia interna-CoBi- nos fue de gran ayuda para intentar identificar los restantes signos, pudiendo con bastante seguridad proponerse una lectura SECoBiRICeA o, mejor aún, SEGoBiRIGeA, dado que su escripción no es incompatible con la práctica escrituraria del patrón binoclusivo o distinción entre oclusivas sordas y sonoras mediante la adición de un trazo adicional para las primeras (una primera aplicación para el celtibérico en Jordán, 2005).

En esta lectura ofrecerían sobre todo alguna duda la antepenúltima y la penúltima letra. La primera letra responde al común tipo de $s$ en forma de abanico, es decir, especialmente estrecha por arriba y ancha por abajo $\langle M\rangle$ solamente que deteriorada en la parte superior a la izquierda del lector; la segunda letra corresponde al sencillo grafo de tres trazos para /e/ $\langle t\rangle$; la forma clásica de reloj de arena encontramos para $<$ Co $>$, esto es, $\langle\nabla\rangle$, forma ambigua para su lectura binoclusiva, pues puede representar la sonora frente a $\langle\mathbb{Z}>$ o bien la sorda frente a la variante sonora del mismo signo y donde faltaría la línea superior horizontal. Sigue la forma para /bi/ ya más bien $\langle\Gamma\rangle$ que $<\Gamma>$. A continuación la forma clásica de globo para la $/ \mathrm{r} /$ celtibérica $\langle\hat{\rangle}\rangle$ no ofrece mayor dificultad y resultaría decididamente muy forzado interpretar este signo como $<\downarrow>$ o $<\mathrm{Be}>$. La grafía que identificamos como /i/, esto es, $\langle\psi<>$ es la de más opaca lectura, séase por la mayor inclinación del ductus hacia la derecha en este 


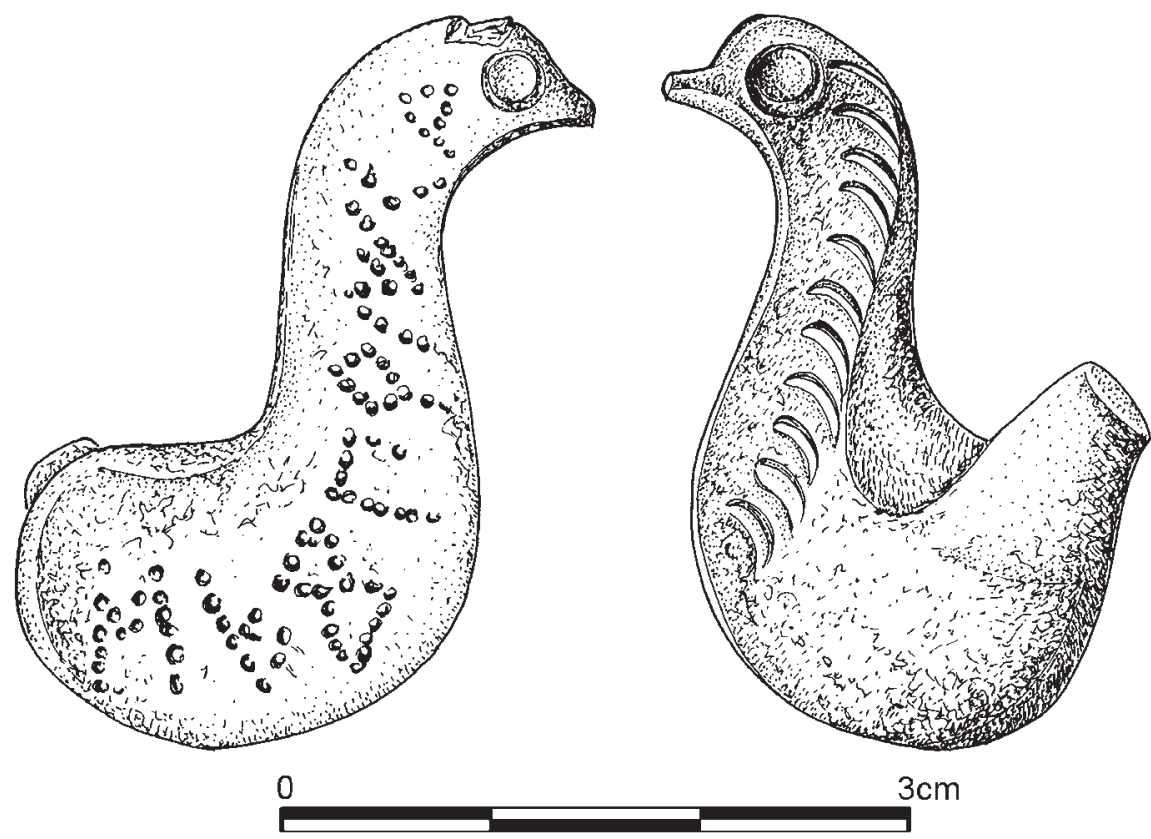

Figura 3: Dibujo a partir de fotografía de la tésera de Torrejoncillo del Rey (dibujo de M. ${ }^{a}$ Dolores Sánchez de Prado).

caso, séase por la aparición de muescas sobre esta letra y que parecen dotarla de una adicional línea horizontal entre las dos hastas laterales y verticales. Además esta letra en su último punto, en la parte superior derecha, es contigua al punto que hace de eje central para la letra siguiente: /ke/ o, mejor, /ge/, pues aparece en su variante más simple de $\langle<$. Bien legible a su vez la última letra: /a/ en su clásica versión de $\langle p\rangle$. Nótese, por tanto, que para notar el grupo de muta cum liquida /bri/ se habría optado por la más habitual fórmula de recurrir a una grafía $<-\mathrm{BiRI}->$ frente a las menos frecuentes fórmulas de $<\mathrm{BiR}>$ o de $<\mathrm{Bi}>$.

\subsection{IMPLICACIONES}

De confirmarse nuestra lectura y la autenticidad de la pieza, esta resulta muy relevante para dejar casi definitivamente tocadas o resueltas algunas cuestiones - minutias curat philologus - de los estudios de Celtiberística tocantes fundamentalmente a otras dos piezas inscritas y a un buen número de cuestiones conexas más o menos directamente con la lectura de estas dichas piezas.

\subsubsection{Para la tésera tauriforme (K.0.3)}

En efecto, la misma e idéntica lectura SECoBiRICeA era ya la aceptada sin reservas por Untermann (1997, 540-542; K.0.3) para una de las caras de una tésera también zoomórfica - tauriforme en concreto- conocida ya por Hübner $(1893,174-175)$ pero de procedencia indeterminada (Hübner 1893, 174: «Unde provenerit ignoratur»), mientras que, por la singularidad del trazo, «que no coincide exactamente con ninguno de los signos celtibéricos regulares» (De Hoz, 1986, 69), proponía Untermann una lectura SECoBiRICeA, es decir, con algunas dudas sobre el penúltimo signo para la otra cara de la pieza, puesto que en concreto esta tésera presenta la particularidad de estar escrita por ambas caras, tanto por la cara plana y en módulo mayor - donde Untermann leería sin dudas SECoBiRICeA, lectura secundada por Almagro-Gorbea $(2003,211)$ - como por la cara en relieve y con un módulo menor para las letras, donde Untermann ya ve menos segura la identificación de $<\mathrm{Ce}>$. El mismo Untermann $(1997,542)$ se hacía eco de las propuestas de otros autores que - para ambas caras - preferían

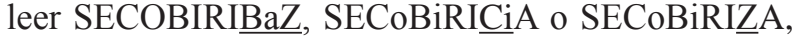
pues, en efecto, parece la hipótesis más sensata la de suponer que las dos caras presentarían siempre, en todo caso, un mismo texto.

Para su propuesta SECoBiRICiA, por cierto, genéricamente señala De $\operatorname{Hoz}(1986,69)$ como analógica «una forma mal trazada en su parte inferior de la variante atestiguada por ejemplo en la escritura monetal de Obulco (A. 100 - A. 102)». Pero obviamente para un signo de lectura problemática en la escritura celtibérica no parece el mejor ni más directo apoyo el de un signo en una escritura que es ya ibérica y en su variante meridional y sinistrorsa. Dicho esto, francamente no hemos podido localizar en la amplia gama de leyendas monetales recogidas por Untermann (1975, 273-283) la forma - y además «mal trazada» $(i ?)$ - en cuestión a la que pudiera referirse De Hoz.

En este estado de cosas los colegas Jordán y Díaz (2006) procedieron a efectuar una autopsia de la tésera 
tauriforme (K.0.3) ofreciendo unas precisas fotografías que mostraban que el problemático signo ofrecía en ambas caras en realidad una fisonomía prácticamente idéntica y singular, fisonomía consistente en una forma $<>>$ que en su parte inferior es prolongada por una pequeña línea recta y vertical, forma que estos autores interpretaron como una variante del habitual sigma celtibérico, idéntico, en efecto, al signo ahora bien legible sólo que prolongado éste por una pequeña línea recta pero diagonal. Con tal propuesta los citados autores venían a secundar, por tanto y mutatis mutandis, la lectura que en su día hiciera Almagro Basch $(1982,203 ; 1984,17)$, propuesta en la que se reafirmaría poco después Jordán $(2007,128)$ : «Esta pieza presenta un lugar difícil de lectura en el séptimo signo de ambas caras [...] creemos que es una $\$$, de modo que la transcripción quedaría en primer lugar como sekobiriza».

\subsubsection{Para un tratamiento fonético en celtibérico}

Yendo, en fin, más lejos Jordán y Díaz (2006), basándose en su nueva lectura y algún otro dato, proponían para la lengua de los celtiberos un tratamiento fonético de palatalización de [g] ante [j], partiendo del supuesto de que su SECoBiRIZA remontaría a una forma adjetival */segobrigia/, id est, *[segobrigja].

Demasiadas hipótesis y demasiados frágiles y escasos los datos para sustentarlas, en opinión de uno de nosotros, que escribía hace unos pocos años: «Si nos parece excelente la idea de revisar la lectura SECoBiRICeA (K.0.3) uel SECoBiRICiA por Jordán y Díaz 2006, ya no podemos estar de acuerdo en que, apoyándose apenas exclusivamente en su relectura SECoBiRIZA, quieran formular aquellos poco menos que una nueva ley fonética para el celtibérico. En la argumentación desplegada se acumularían otros problemas [...] sigue abierta la posibilidad de que otro texto con un SECoBiRICeA (Fletcher y Pérez 1994) sea auténtico y $[\ldots]$ está sobre todo para nosotros el obstáculo de que seguimos viendo $\langle\mathrm{Ce}>$ o mejor $<\mathrm{Ge}>$, ya que el texto estaría escrito en patrón binoclusivo: SEGoBiRIGeA. De modo que, cuando tras la aportación de Rodríguez 2001-2002 — aceptada por el mismo Jordán 2008, 124- creíamos confirmada la gran variedad alográfica para el silabemograma $<\mathrm{Ce}>$, resulta que Jordán y Díaz 2006 leen SECoBiRIZA y con ello postulan un tratamiento para [gj] tras /i/ o tras otra vocal en celtibérico (2006, 136-137). El error metodológico está, en nuestra modesta opinión, en sustentar una formulación general en apenas una única y no segura lectura».

\subsubsection{Para las formaciones adjetivales}

Por otra parte, nos parece que precisamente la presencia, en la pieza tauriforme, de la singular variante antes comentada para el específico valor sonoro $<\mathrm{Go}>$ mediante el signo de «mesa plegable invertida» —es decir, el signo $\langle\nabla\rangle$ pero desprovisto de su línea horizontal superior - supone un refrendo a la intencionada escripción binoclusiva del texto, lo que ayudaría a explicar también la singular forma para $<\mathrm{Ge}>$. Como daño colateral este documento socava además el principal apoyo a la problemática hipótesis fonética * [igj > z] o * [i/ugj > z] de los mismos Jordán y Díaz (2006, 137), basada necesariamente, como vimos, en la aceptación de una formación derivativa original */ segobrigia/, id est, *[segobrigja], aunque, naturalmente, caben otras posibilidades, algunas ya señaladas por diversos autores, cuales */segobrigeia/ (uide Wodtko, $2000,328)$ o incluso una */segobrigaia/, sufijo que quizá podría estar también en el LESTERAIA del título del tercer bronce de Botorrita (K.1.3), en MUCuUCaAIU (K.13.2), NEMAIOS (K.14.1 A), NEMAIOSO (K.14,2), SEECeEIOS (K.24.1), SICeIA (K.1.3 II-47), TRECAIAS (K.3.3 -3) o en el etnónimo de los vacceos (Plin. nat. 3,4,19: Vaccæi; Ptol. geogr. 2,6,49: Ovंaккаïl etc.).

En todo caso, en razón de la analogía contextual, sí cabe interpretar el término —al menos etimológicamente - como una formación adjetival y así ha venido siendo tradicionalmente aceptado y con buenos argumentos ya desde Lejeune (1955, 45, 83 y 103; uide ítem Wodtko, 2000, 328), aunque dicha formación resulte aislada o al menos muy minoritaria frente a la en celtibérico habitualísima y regular derivación con $-k-$. Para explicar el surgimiento de tal formación alternativa De $\operatorname{Hoz}(1986,69)$ propuso que esta podría venir condicionada por la intención de evitar «una excesiva acumulación de oclusivas velares», para evitar, pues, una doble secuencia gutural: **/segobrigika/ o incluso **/segobrigaka/, formaciones en principio esperables y que, pese a la altísima frecuencia de los topónimos en -briga, no han sido, sin embargo, hasta ahora documentadas para dicha específica y polisilábica forma, aunque sí parecería acreditada al menos la marca $-k$ - en casos cuales Brigaikinôn Brigaíkion entre los

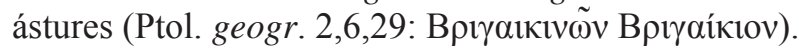
Nótese que el topónimo ptolemaico estaría directa o indirectamente corroborado por otras fuentes: Brigcecini (Flor. epit. 2,33,1); Briceco y Brigeco (It. Ant. 439.8 y 440.1 respectivamente); BRIGECIO (It. Ast. 2.3); Brigicon (It. Rau. 319.1); BRIGEACIS (C.I.L. 2,63381); BRIGIAECINO (C.I.L: 2,6094) o AIIOBRIGIAECINOS (bis) en el edicto del Bierzo (Balboa 1999). En fin, tampoco puede excluirse taxativamente que, tal como parece, el topónimo Brihuega, en Guadalajara, tenga su origen en un derivado mediante $-k-$ del tan ubicuo -briga hispánico. De hecho, ya Villar y Untermann $(1999,724)$ esgrimían contra la teoría preventiva de velares apuntada por De Hoz una forma como BRIGEACIS (C.I.L. 2,63381) concluyendo que no existía base empírica para suponer que SECoBiRICeA o un CaTeRAICiNA — en la denominada Tésera Turiel 1- «deban su sufijo -ino- en vez de -ko-a una 
especie de disimilación (preventiva o no) tendente a evitar la acumalación de velares».

Como fuere, aquella secuencia ptolemaica (geogr. 2,6,29) Brigaikinôn Brigaíkion entre los ástures resultaría interesantísima por documentar otra marginal formación adjetival celtibérica /-aikin-/, analizable como una formación compuesta /-aik-in-/ con /-in-/, al menos en este caso, como suplemento a su vez adjetival de una presumible forma adjetival /-aik-/, lo que supondría que las análogas formaciones CaTeRAICiNA (Tésera Turiel 1) y RoUTaICiNA (en la tésera de la denominada Colección Pellicer 8; Almagro-Gorbea 2003, 389-390 y 2004, 346-347, con superación de análisis metalográfico) podrían remitir a sendos *CaTeRAICiON y *RoUTaICiON, formaciones que, por tanto, a su vez podrían ser derivados - como supuestamente el ptolemaico Brigaíkion de un *Briga, de *CaTeRA y *RoUTa respectivamente (para la segunda forma

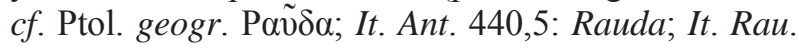
312,15: Rodacis) - en neutro con posible valor diminutivo, como apuntamos en otro lugar. Como prueba de un segmento -aik-, en concreto, en su catálogo de formaciones adjetivales en $-k$ - Villar y Jordán (2001, 173 y 179) recogen la forma CuSTaICoS (K.1.1. A-7), fácilmente relacionable, en efecto, con el prácticamente contiguo CuSTa (K.1.1. A-5), como ven los citados autores, lo que asimismo sería compatible con la hipótesis de una secuencia $*[-a>-$ aik->-aikin-]. Si la lectura es correcta, la forma $S A I B O D A E / C I$ de una epígrafe vallisoletana y dada a conocer recientemente (De Bernardo y Sanz, 2009) podría contener la citada secuencia adjetival $* /$-aik-/ (para posibles formas $A N$ DAIECO, ASSAECO y BARCIAECO con */-aik-/ uide Prósper, 2002, 325-328). Un sufijo -in-, por otra parte, reconocerían también Villar y Untermann $(1999,723)$ en el caso al menos de TeCaMETiNAS (K.1.1,A-8) y TeRCiNOS (K.1.3 passim).

\subsubsection{Para el origen de la pieza}

Por otra parte, la tésera tauriforme con la doble inscripción SECoBiRICeA (K.0.3) ha sido tradicionalmente tenida como de origen desconocido y ya Almagro senior $(1982,204)$ aclaró en su día las razones de la errónea atribución a una procedencia - alguna vez señalada - en Huete (Cuenca). Más recientemente Burillo $(2008,406)$ ha aportado un dato historiográfico de sumo interés y que, sin embargo, había pasado desapercibido a los investigadores, pues Bernardino Martín Mínguez $(1883,196)$ habla presumiblemente de esta pieza - «una preciosa media cabeza de toro con la misma leyenda»-como perteneciente a un coleccionista privado de Valladolid y además refiere la intención del propio Martín Mínguez de interceder para que la pieza sea donada «al Museo arqueológico». Aunque Martín no especifica el singular detalle de que esa «misma leyenda» se encuentra en ambas caras y aunque con tal expresión debe de referirse además a la lectura
SECoBiRICeZ que se da en una moneda (A.89), lectura de la que, por tanto, sólo se diferenciaría en el último signo, difícilmente puede tratarse, en efecto, de una pieza distinta de la que contiene el doble SECoBiRI$\mathrm{CeA}$. Ahora bien, la afirmación categórica de Burillo $(2008,406)$ : «Estas vinculaciones con el entorno de Segóbriga deben desecharse de forma definitiva», ha de tomarse con alguna prevención, ya que obviamente su localización en manos de un coleccionista y en la ciudad de Valladolid no comporta necesariamente que la pieza procediera de esta misma provincia.

\subsubsection{Para una lámina de plomo}

A favor de aquella misma lectura de SECoBiRICeA para la tésera tauriforme estaba también la aparición de un nada ambiguo SECoBiRICeA (MEZPQR (D) en una lámina de plomo, donde la inequívoca forma $<\mathbb{}>$ para $<\mathrm{Ce}>$ aparecía como correspondiente al problemático signo penúltimo de la citada tésera. La pieza de ignota procedencia y conocida mucho más recientemente - editada en 1994 por Fletcher y Pérez- debería presumiblemente haber zanjado la cuestión a favor de una lectura SECoBiRICeA para la tésera; sin embargo, sólo mantuvo su solución — podría decirseen suspenso, ya que la pieza fue considerada falsa por Untermann $(1997,355)$.

Con la nueva propuesta de lectura para la tésera tauriforme, por su parte, Jordán y Díaz $(2006,135)$ creyeron que ahora quedaba apoyada «la suposición de falsedad de la lámina donde se lee sekobirikea, por parte de J. Untermann». Si antes en el caso de la lámina su importancia radicaba, como reconociera Jordán (2004, 294), «en que podría confirmar la lectura» de la tésera tauriforme, ahora se pasaba la capacidad decisoria al bando contrario y la nueva lectura apoyaba la suposición de falsedad de la lámina, cuyo falsario, por tanto, habría seguido la tradicional interpretación - errónea para Jordán y Díaz- de la tésera.

Este nuevo texto -insistamos: en el caso de confirmarse la lectura aquí propuesta y la autenticidad de la pieza - refrendaría la unánime lectura SECoBiRICeA siue SEGoBiRIGeA —en definitiva: /segobrigea/para los tres textos en las dos piezas señaladas, apoyando ahora, por tanto, la autenticidad de la lámina de plomo. Además una de las cuatro objeciones que Untermann $(1997,355)$ esgrimía contra la autenticidad de esta pie$\mathrm{za}$, a saber, el ser hasta entonces la única inscripción celtibérica sobre plomo («bis jetzt einzige keltiber. Inschrift auf Blei»), ha quedado superada por la aparición de «La primera inscripción celtibérica sobre plomo» editada por Lorrio y Velaza (2005), pero que ahora ya no sería exactamente «la primera», si bien ha de recordarse que aquella misma lámina contenía en su otra cara una inscripción en lengua ibérica (BeCuENBeTiLIŘE), hecho doblemente objetado ibidem por Untermann en razón de la presencia de la vibrante no celtibérica $\breve{R}$ y la estructura no celtibérica del texto, aspectos empero 
ambos explicables si, como se acaba de decir, se considera ibérico y no celtibérico el texto de dicha otra cara. La cuarta objeción untermanniana: el considerar SeCoBiRiCeA la «copia de una conocida inscripción», la de la citada tésera tauriforme (K.0.3), quedaría ahora como una petición de principio.

\subsubsection{Para la leyenda monetal SECoBiRiCeZ (A.89)}

También, como estamos viendo, inevitablemente, a causa de su contenido, esta tésera presentaría relación no sólo con la tésera tauromorfa SECoBiRICeA (K.0.3) o con la lámina con igual texto, sino también y obviamente con la monetal SECoBiRICeZ (A.89). Inicialmente, autores de tanta relevancia como Jürgen Untermann (1997, 542: «Segobrig-s (latinisiert zu Segobriga), bezeugt durch die Münzlegenden sekobirikeđ, SEGOBRIS und SEGOBRIGA») y De Hoz (1986, 51 : «posiblemente debemos leer /segobriks/») postularon que dicha forma debía entenderse con un nominativo singular realizado /segobrigs/ o /segobriks/, de modo que en el segmento gráfico final $<\mathrm{CeZ}>$ la vocal era muda y su presencia obligada por la escritura silabemográfica para las consonantes oclusivas. En prueba de ello podía aducirse y se aducía, como acabamos de ver, una leyenda monetal SEGOBRIS de la que explícitamente García-Bellido y Blázquez (2001: II 340 n144) dicen: «Emisión intermedia con el nombre celtibérico Segobrix escrito en latín SEGOBRIS. Su único hallazgo conocido en Uxama (Soria) haría suponer que todavía se acuña en la Meseta Norte».

Igualmente en relación a SECoBiRICeZ GarcíaBellido desde hace ya unos años (1974, 382-386; 1993,$210 ; 1994 \ldots$ ) ha venido insistiendo con sólidos argumentos en la «localización en la mesopotamia del Duero y el Pisuerga para la ceca» (2007, 208-210), sosteniendo la posibilidad de que tras las guerras sertorianas se trasladase la ciudad desde la Meseta Norte a Cabeza de Griego, ocasionando este nuevo e importante emplazamiento el declive o final de la cercana Contrebia Carbica: «esa ciudad republicana de la Celtiberia norte, quizás fuese arrasada por los romanos tras las guerras sertorianas o durante ellas, y [...] trasladada su población al enclave que hoy conocemos en Cabeza de Griego» (García-Bellido,1994, 246), de modo que la Segobriga conquense «debió de ser fundada con el traslado de los habitantes de la Segobrix republicana» (García-Bellido,1994, 258). En su día Sacristán (1994, 145) señaló además «el alto de San Pedro, en Pinilla Trasmonte», un gran oppidum con una extensión de cerca de 18 hectáreas como posible sede de la antigua *Segobrix o Segobris en territorio burgalés.

\subsubsection{Para el caso flexivo de SECoBiRiCeZ}

Más recientemente, sin embargo, parece haberse asentado la idea de que en SECoBiRICeZ no tengamos un nominativo sino un ablativo. Así y haciéndose eco de los argumentos a favor de ello expuestos por De Bernardo (1996, 239-240) ya Villar (1995, 25; secundado por Jordán 2004, 125 y 134) veía en SECoBiRICeZ un ablativo - de un tema en consonante - con $<\mathrm{e}>$, por tanto, no muda sino bien sonora en la última sílaba. Ciertamente para un nominativo /segobrix/ se hubiese esperado, para empezar, más bien la otra silbante: **SECoBiRICeS, pese a la observación de De Hoz $(1986,51)$ de que posiblemente la utilización de $Z$ en vez de $\mathrm{S}$ «en este caso se deba al azar».

Otra cuestión sobre las que mantenemos nuestras dudas es si por mor, entre otras razones, de su frecuencia y los contextos donde aparecen, no cabría plantearse la posibilidad de que, aun manteniendo y reconociendo el origen etimológico en el ablativo, estas formas no masculinas en $-Z$ en realidad fueran funcionalmente genitivos, al menos en el caso de las formas no antroponímicas.

\subsubsection{Para la duda entre /-briks/ y /-bris/}

En todo caso, como vemos, el reconocimiento de SECoBiRICeZ como ablativo pero siempre de un tema en consonante -id est: no con un nominativo Segobriga, para el que el etimológico ablativo esperable tendría una desinencia $-\mathrm{AZ}$ en escritura celtibérica - permite seguir manteniendo la hipótesis de un nominativo */ segobri[k]s/ para dicho ablativo sólo que no documentado... ¿o quizá sí? Aunque el detalle no es trascendente tanto la efectiva documentación de un SEGOBRIS cuanto la general e inveterada tendencia mesetaria u occidental a simplificar los grupos consonánticos explosivos, especialmente en posición átona — baste aducir el AMBATO de la tésera de Ubierna (Castellano y Gimeno 1999, 359-360) frente al conocido ambactus latino (Cæs. Gall. 6,15,2: ambactos) — hacen más sólida la hipótesis de que en la pronunciación habitual el elemento velar ya se había perdido y la pronunciación efectiva era en /-is/ y no en /-iks/ o incluso /-igs/, pérdida que no impedía, por supuesto, el mantenimiento de la velar en posición intervocálica en otros casos de la flexión, como sin más documentaría SECoBiRICeZ, tal como, por ejemplo, la pérdida de la $-n$ final en los nominativos latinos leo u homo no impide su conservación en otros casos (leonis, hominem...). Así también para Jordán $(2004,193)$ mientras SECoBiRICeZ «no deja lugar a dudas de su clasificación como un tema en velar, una forma en epigrafía latina SEGOBRIS apunta a una realización ya [segobris]».

\subsubsection{Para el doblete de -bris y -briga}

A favor de un nominativo en -is estaría también la documentación toponímica más occidental, especialmente en la zona galaica, donde ya hace tiempo se notó la presencia relativamente frecuente de topónimos 
terminados en -bri[s], cuales ERCORIOBRI (C.I.L. 2,2711), el verosímilmente castellum de MIOBRI del epitafio de Crecente (Lugo; Rodríguez 1999), la también galaica Phlaouía Lambrís (Ptol. geogr. 2,6,26: $\Phi \lambda \alpha$ ovía $\Lambda \alpha \mu \beta \rho i ́ s ; c f$. el topónimo coruñés Lambre), o el derivativo BLANIOBRENSI o ELANIOBRENSI de Astorga (León; C.I.L. 2,5667). Asimismo probablemente la mayoría de los numerosos microtopónimos gallegos en -bre remontaría[n] a un antiguo final en -bris antes que en -briga: Alxibre, Anzobre, Baiobre, Barallobre, Bembibre, Canzobre, Cecebre, Cezobre, Coebre, Ixobre, Laxobre, Lestrobe, Maiobre, Pantiñobre, Rañobre, Sebre...

Esta suma de testimonios apoyaría, por tanto, la pronunciación efectiva ya en época histórica en $/-$ is/ y no en /-iks/, tal como fehacientemente documentaría SEGOBRIS. Frente a esto y a favor de una final en /iks/ apenas sí contamos con formas como Kaitóbrix

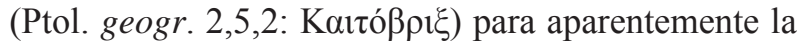
misma ciudad que Mela $(3,1,6)$ da como Caetobriga y donde además la forma final $-1 \xi$ puede deberse a la helenización lingüística llevada a cabo por los primeros geógrafos griegos, adaptaciones especialmente frecuentes para poblaciones conocidas desde antiguo o importantes, como era el caso de esta ciudad que se identifica con Setúbal, topónimo que a su vez se dejaría explicar algo mejor desde una final en -briga que desde un final en -brix.

Ahora bien, aunque pertenecientes a la misma raíz, acaso -bris y -briga hayan significado entidades algo distintas en su momento, quedando -bris como denominación para referentes menores como castella y briga para entidades urbanas mayores.

Así las cosas, en la antigua doble hipótesis de García-Bellido (1993, 210): «Puede tratarse de dos ciudades homóninas, o de una misma entidad política trasladada desde Celtiberia a Carpetania después de las guerras sertorianas», no sólo recibe más argumentos la segunda, sino que más probablemente se trate de dos ciudades no homónimas una, la burgalesa-arevaca, con nominativo Segobris —o más hipotéticamente *Segobrix - y otra, la conquense-carpetana, con nominativo Segobriga. De modo que, aunque, como vimos, se ha apuntado a veces que la forma Segobriga sería el resultado de la latinización de un /segobri[k]s/ (Untermann, 1997, 542: «latinisiert zu Segobriga»), en realidad SEGOBRIS y SEGOBRIGA deben de responder a formas indígenas con esencialmente igual base etimológica pero matices o connotaciones diferentes ( $c f$. quizá los latinos castellum - castrum o los Castil y Castillo o Villa y Villar de la toponimia española). Para la morfología latina, por otra parte, eran perfectamente aceptables tanto los nominativos en $-i s$ como en $-i x$.

\subsection{RECAPITULACIÓN DE LAS PRINCIPALES IMPLICA- CIONES}

Momento para una recapitulación final pero necesariamente provisional:

- SECoBiRICeA - y no *SECoBiRICiA o *SECoBiRIZA - sería la lectura correcta de la tésera tauriforme (K.0.3).

- Las objeciones de ser copia de un texto conocido y en soporte de plomo ya no serían tan válidas para mantener la falsedad de la lámina con SeCoBiRICeA editada por Fletcher y Pérez (1994).

- En tal caso, el plomo celtibérico que podría «proceder, según noticias sin confirmar, de un yacimiento de la zona de Castillejo de Iniesta» (Lorrio y Velaza, 2005, 1032) ya no sería «La primera inscripción celtibérica sobre plomo» conocida sino la lámina con SECoBiRICeA.

- El origen, pace Burillo (2008, 406), en el alto Duero de la tésera tauriforme rezando SECoBiRICeA no es segura.

- La tradicional relación entre la altoduriense SECoBiRICeZ y al menos está prácticamente segura conquense SECoBiRICeA seguiría, no obstante, siendo mantenible en los términos de trasvase de población ya anunciados por García-Bellido (1993, 1994, 2007...; Burillo, 2008, 405-412).

- La probable inexistencia de un *SECoBiRIZA debilita seriamente la propuesta de ley fonética efectuada en su día por Jordán y Díaz (2006).

- Fuera cual fuera su concreta etimología, quedaría confirmada la existencia de una derivación adjetival en -E- en la lengua de los celtiberos, formación quizá documentable también en otras formas.

- SeCoBiRICeZ no representa un nominativo en -is o en $-i x$ sino una forma de ablativo etimológico quizá empleado como genitivo.

- Hay más argumentos a favor de un nominativo fonéticamente /-bris/ que a favor de /-briks/.

- SECoBiRICeZ pertenecería a la flexión de SEGOBRIS y no a la de Segobriga.

- Los componentes -briga y -bris podrían indicar referentes algo distintos.

No es mucho, pero tampoco es poco para un texto tan breve.

Xaverio Ballester

Max Turiel

Dpto. Filología Clásica

Facultad de Filología

Avda. Blasco Ibáñez, 32

46010 Valencia 


\section{BIBLIOGRAFÍA}

Almagro BASCH, M., 1982: «Tres téseras celtibéricas de bronce de la región de Segóbriga. Saelices (Cuenca)», En homenaje a Conchita Fernández Chicarro: Directora del Museo Arqueológico de Sevilla, 195-209, Madrid.

Almagro BASCH, M., 1984: Segobriga II. Inscripciones ibéricas, latinas paganas y latinas cristianas, Madrid.

Almagro-GorbeA, M., 2003: Epigrafía Prerromana, Madrid.

Almagro-Gorbea, M., CAsado, D., Fontes, F., MedeROS, A. y TORReS, M., 2004: Prehistoria. Antigüedades españolas I, Madrid.

Almagro-Gorbea, M. y ABAscal, J. M., 1999: Segóbriga y su conjunto arqueológico, Madrid.

Almagro-GorbeA, M. y LORRIO, A. J., 2006-2007: «De Sego a Augusto: los orígenes celtibéricos de Segobriga», BSAA. Arqueología, 72/73, 143-181.

BAlBoA DE PAZ, J. A., 1999: «Un edicto del emperador Augusto hallado en el Bierzo», Estudios Bercianos, 25, $45-53$.

BurIllo, F., 2008: Los Celtíberos. Etnias y estados, Madrid.

Castellano, Á. y Gimeno, H., 1999: «Tres documentos de hospitium inéditos», en F. VILLAR y F. BELTRÁN (eds.), Pueblos, Lenguas y Escrituras en la Hispania Prerromana, 359-374, Salamanca.

De Bernardo StemPel, P., 1996: «Die Stummvokale: eine Bilanz für das Keltiberische», en W. MEID y P. ANREITER (eds.), Die grösseren altkeltischen Sprachdenkmäler. Akten des Kolloquiums Innsbruck, 2. April-3. Mai 1993, 212-256, Innsbruck.

De Bernardo Stempel, P. y Sanz Mínguez, C., 2009: «Nueva estela funeraria romana con onomástica céltica, de Padilla de Duero (Peñafiel, Valladolid)», BSAA arqueología, 75, 223-242.

DE Hoz, J., 1986: «La epigrafía celtibérica», Epigrafía hispánica de época romano-republicana, 43-102, Zaragoza.

Fletcher VAlls, D. y PÉREZ VILATEla, L., 1994: «Dos textos celtibéricos de procedencia desconocida», Archivo de Prehistoria Levantina, 21, 357-366.

GARCÍA-Bellido, M. P., 1974: «Tesorillo salmantino de denarios ibéricos», Zephyrus, 25, 379-395.

GARCÍA-Bellido, M. P., 1993: «Sekobirikes», Tabula Imperii Romani. Hoja K-30: Madrid. Caesaraugusta. Clunia, 210

GARCÍA-Bellido, M. P., 1994: «Sobre la localización de Segobrix y las monedas del yacimiento de Clunia», Archivo Español de Arqueología, 67, 245-259.

GARCÍA-BELLIDO, M. P., 2007: «Numismática y territorios étnicos en la meseta meridional», G. Carrasco coord., Los pueblos prerromanos en Castilla-La Mancha, 199226, Cuenca

GarcíA-Bellido, M. P. y BlÁzQuez, C., 2001: Diccionario de cecas y pueblos hispánicos, II vols, Madrid.
HÜBNER, E., 1893: Monumenta linguae Ibericae, Berlín.

JORDÁN CÓlERA, C., 2004: Celtibérico, Zaragoza.

JORDÁN CÓLERA, C., 2005: «¿Sistema dual de escritura en celtibérico?», Palaeohispanica, 5, 1013-1030.

JORDÁN CÓLERA, C., 2007: «Estudios sobre el sistema dual de escritura en epigrafía no monetal celtibérica», $\mathrm{Pa}$ laeohispanica, 7, 101-142.

JORDÁN CÓLERA, C., 2008: «Toponimia y Etnonimia en leyendas monetales Celtibéricas y Vasconas: 1. tarmeskom NO bormeskon. 2. bolśken NO bolśkan», en J.L. GARCÍA (ed.), Celtic and Other Languages in Ancient Europe, 119-132, Salamanca.

JORDÁN CÓLERA, C. y DÍAZ, B., 2006: «[K.0.3] Ni sekobirikia ni sekobirikea: sekobiriza. A propósito del tratamiento ${ }^{* g-y o d ~ e n ~ c e l t i b e ́ r i c o », ~ P a l a e o h i s p a n i c a, ~ 6, ~}$ $131-138$

LEJeUne, M., 1955: Celtiberica, Salamanca.

LORRIO, A. J. y VELAZA J., 2005: «La primera inscripción celtibérica sobre plomo», Palaeohispanica, 5, 1031-1048.

Martín MíngueZ, B., 1883: Datos Epigráficos y Numismásticos de España, Valladolid.

PRÓSPER, B. M., 2002: Lenguas y religiones prerromanas del occidente de la Península Ibérica, Salamanca.

Rodríguez COLMENERO, A., 1999: «Sobre dos nuevas estelas monumentales de Lucus Augusti», en F. VILLAR y F. Beltrán (eds.), Pueblos, Lenguas y Escrituras en la Hispania Prerromana, 605-617, Salamanca.

RODRÍGUEZ RAMOS, J., 2001-2002: «Okelakom, Sekeida, Bolśken», Kalathos, 20/1, 429-434.

SACRISTÁN DE LAMA, J. D., 1994: "Clunia», Leyenda y arqueología de las ciudades prerromanas de la península ibérica. Ciclo de conferencias. Madrid, 25 y 26 de Noviembre de 1993, II 135-147, Madrid.

SÁNCHEZ-PALENCIA, J., 1993: «Torrejoncillo del Rey», Tabula Imperii Romani. Hoja K-30: Madrid. Caesaraugusta. Clunia, 224.

UNTERMANN, J., 1975: Monumenta Linguarum Hispanicarum. Band I. Die Münzlegenden. 2. Tafeln, Wiesbaden.

UntermanN, J., 1997: Monumenta Linguarum Hispanicarum. Band IV. Die tartessischen, keltiberischen und lusitanischen Inschriften, Wiesbaden.

VILLAR, F., 1995: Estudios de celtibérico y de toponimia prerromana, Salamanca.

Villar, F., Díaz, M. A., Medrano, M. M. y Jordán, C., 2001: El IV Bronce de Botorrita (Contrebia Belaisca): Arqueología y Lingüística, Salamanca.

VILlAR, F. y UNTERMANN, J., 1999: «Las "téseras” de Gadir y Tarvodurum», en F. VILlar y F. BELTRÁN (eds.), Pueblos, Lenguas y Escrituras en la Hispania Prerromana, 719-731, Salamanca.

WodtKo, D. S., 2000: Monumenta linguarum Hispanicarum. Band V.1. Wörterbuch der keltiberischen Inschriften, Wiesbaden. 